\title{
The earth dynamic system: the earth rotation vs mantle convection*
}

\author{
Shuping Chen ${ }^{1,2}$ \\ ${ }^{1}$ State Key Laboratory of Petroleum Resource and Prospecting, China University of Petroleum, Beijing, China; \\ ${ }^{2}$ College of Geosciences, China University of Petroleum, Beijing, China; csp21c@163.com
}

Received 18 September 2010; revised 20 October 2010; accepted 25 October 2010.

\begin{abstract}
The earth dynamic system is one of the key scientific questions on the earth science. The thermodynamic behavior and gravity force of the earth and the rheology nature of the mantle prove that mantle convection is the main power source leading the lithosphere to break and move. Yet the directivity of both the structures in the crust and plate movement reminds of the earth rotation. Here we demonstrate that the mantle convection and inertia force of the earth rotation affect each other, the former being the power source of lithosphere plate break and motion, and the latter determining the direction of the mantle convection and plate motion. The sense of plate motion depends on the mantle upwells, whose trends are controlled by the earth rotation. The geometric shapes of the plate boundaries can adjust the direction of plate movement.
\end{abstract}

Keywords: Plate Tectonics; Earth Rotation; Mantle Convection; Global Dynamics

\section{INTRODUCTION}

The earth dynamic process has long been a subject for debate. Tectonic deformation, such as folds and faults, is a ubiquitous appearance on the Earth's surface. What force leads the rock in the Earth to deform? On the other hand, all structures present orientations either in latitudinal direction, or in longitude direction, or in oblique direction [1-3]. The present mid-oceanic ridges occur along longitude or latitude (Figure 1). The transform faults have good orientation (Figure 2). The flow lines of the lithosphere plates are parallel to the transform faults [4-8]. The plate motions also are in certain direc-

\footnotetext{
*This study is financially supported by the National Natural Science Foundation of China (No.90814007)
}

tions [4]. The questions are where the dynamic force comes from and what element controls the directivity.

Several hypotheses of the earth dynamics once emerged in the earth science, such as the earth expanding hypothesis, the earth contracting hypothesis, the earth pulsing hypothesis, the earth rotation angular speed alternating hypothesis, mantle convection hypothesis, et al. [8]. The plate tectonics hold the mantle convection to be the basic dynamic force of plate movement [9-13], but it does not touch what controls the direction of plate movement or deformation direction.

As early as 1910s and 1920s, Wegener considered the forces related to the earth rotation are the dynamics responsible for the continental drift [14]). Also, the theory of geomechanics thought the extraneous forces related to the change of the earth rotation speed to be the dynamics of the crust motion $[1,2]$, which resolves the question about orientation of tectonic deformation, but faces with the problem on the force magnitude. The change in the earth rotation speed and deformations related to the earth rotation has long been noticed [15-19]. The intensive adjustments of the plates are in sound correspondence to the changes of the earth rotation speed $[12,20]$. The mantle flows along the latitude and longitude, and combined latitudinal and longitudinal mantle flow exists [21]. The relationships among the mantle convection, the earth rotation, the plate motion and the crust rock deformation are worth of insight.

This paper will discuss the relationship between the mantle convection and the earth rotation in controlling the plate motion and rock deformation, based on the two facts of the Earth, the earth's heat and rotation. And a carton model was established.

\section{LAYER STRUCTURE OF THE EARTH}

The Earth is of a layer structure, the crust, the mantle, and the core according to their component (Figure 3). The mantle can be divided into upper mantle, transition zone and lower mantle. The uppermost part of the mantle 


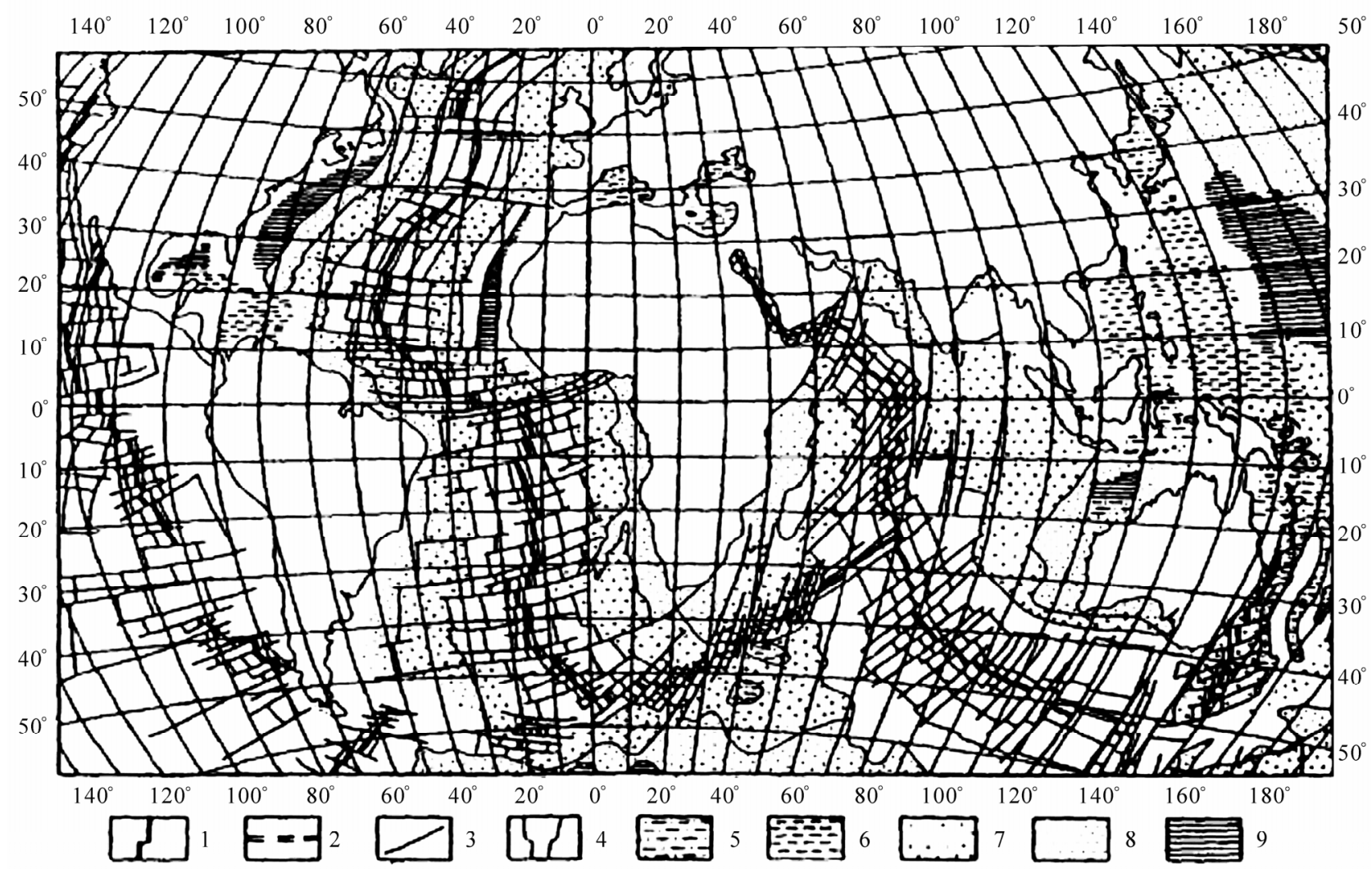

Figure 1. Present distribution of mid-oceanic ridges. 1-longitudinal mid-oceanic ridge; 2-mid-oceanic ridge circling Antarctica; 3-transform fault; 4-magnetic anomaly belts; 5-Oligocene-Neogene; 6-Paleocene-Eocene; 7-late Cretaceous; 8-early Cretaceous; 9-Jurassic. (cited in reference [4]).

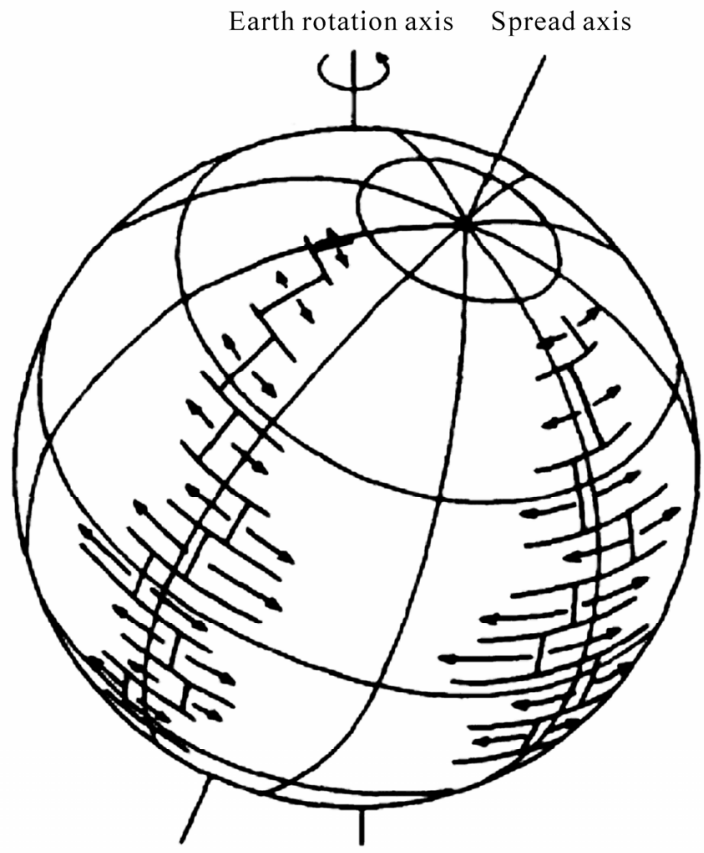

Figure 2. Relationship of transform faults to the Earth rotation. (cited in reference [22]). is rheologically similar with the crust, and they make up the lithosphere, which is cut into plates by deep faults. The earth mantle under lithosphere has less intensity than the lithosphere and its deformation mechanism is flowage. The substance of the mantle is a mixture of the solid and liquid matter, being very pliable and easily coming to plastic flow. So it is called the asthenosphere. Lithosphere can drift above the asthenosphere.

\section{MANTLE CONVECTION}

As a thermal system, the earth is in a course of continual cooling from the beginning of the earth to the present [23]. Heat conduction and convection are its cooling methods. The former mainly occurs in lithosphere and the latter in mantle [24]. The heat convection of the earth interior have two methods which are plate means and mantle plume means [23,25] (Figure 4). As reckoned, the Rayleigh number of the mantle is at least $3 \times 10^{6}$, which exceeds 2000 , the critical value of convection far away. If buoyancy is greater than viscosity resistance, flowage will happen.

In the earth system, the mantle with high density because of cooling or phase transition can flow downward, and 


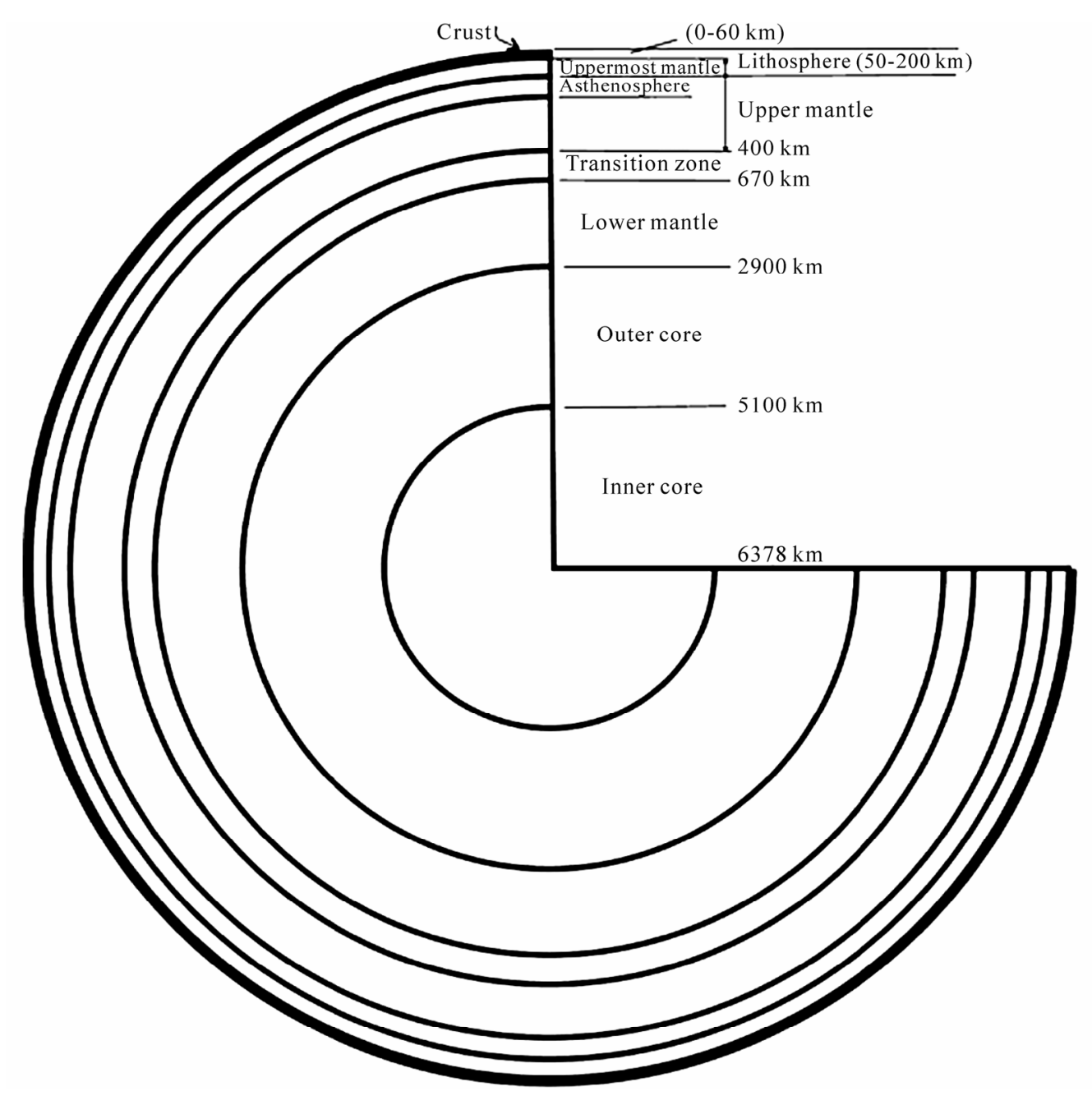

Figure 3. Layer structure of the earth. Not to scale.

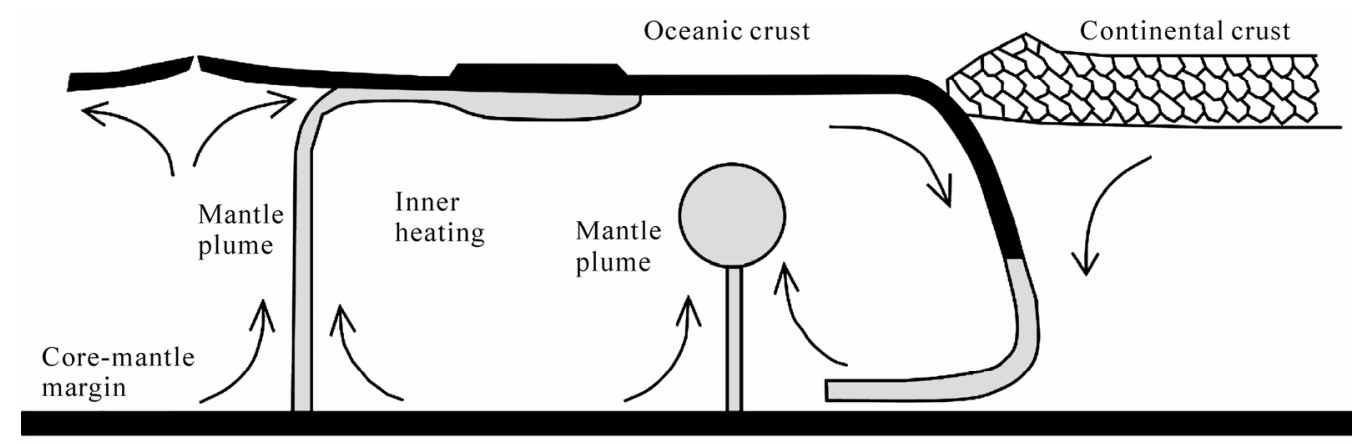

Figure 4. Main components of the mantle's dynamic system (Modified after reference [23]).

the mantle with low density caused by thermal expansion can flow up. According to conservation of substance, flowage in whatever direction should cause flowage in opposite direction, forming convection circles, namely the mantle convection. This is the power source of the lithosphere plate break and movement, and the recognized forces moving the plates are ridge push, slab pull, trench suction and basal drag [26].

\section{FORCE ASSOCIATED WITH THE EARTH ROTATION}

The change in the earth rotation speed has long been found [15]. Force relating to the earth rotation includes 
centrifugal inertia force, latitudinal extraneous force, Coriolis force and so on [20].

When angular rotation speed of the earth changes, an extraneous centrifugal force develops with constituents both in the radial direction F2 and in the longitudinal direction F1 (Eq.1) [20] (Figure 5).

$$
F 1=\frac{1}{2} m(2 w+\Delta w) \Delta w \cdot R \cdot \sin 2 \alpha
$$

where $m$ is the quality of certain unit, $w$ is angular velocity, $\Delta w$ is the increase of the angular velocity, $R$ is the radius of the earth, and $\alpha$ is latitude.

For a terrace with a unit volume, the F1 is as following

$$
F 1=\frac{1}{2} \rho(2 w+\Delta w) \Delta w \cdot R \cdot \sin 2 \alpha
$$

where the $\rho$ is density. For a homogeneous globe, the F1 has maximum value near the latitude of $45^{\circ}$ and decreases to zero toward the poles and the equator (Figure 6). There will be action force between two terraces with different mass or different density, based on Eq.1 and Eq.2.

Meanwhile, due to the earth is an ellipse, the gravity has a component in the radial direction P2 and another constituent in the longitudinal direction P1 (Figure 5). It can be supposed that there is a state where balances exist between the F1 and P1 and between the F2 and the P2. In this case, a terrace in the earth surface is static. When there is change in the earth rotation speed, either the component in radial direction or the component in the longitude will change and the balances will be broken, and vibrations in the radial direction and movements along the longitudinal direction will occur.

On the other hand, change of the earth rotation angular speed may cause force in latitudinal direction F3 (Figure 5) (Eq.3), arousing movement in latitudinal direction. The maximum of the F3 is at the equator of the earth, and decrease toward the poles. It has been proven that the earth rotation angular speed is continually changing during the earth evolutionary process [20], while the main reason that causes the earth rotation angular speed change is the earth shrinking or distending and redistribution of its interior substance, such as mantle upwelling or plate subduction.

$$
F 3=m(\tau-1) \frac{d w}{d t} \cdot R \cdot \cos \alpha
$$

Where $m$ is the quality of certain unit, $\tau$ is an coefficient related to the cohesion between the unit and its basement, $\frac{d w}{d t}$ is the angular acceleration, $R$ is the radius of the earth, and $\alpha$ is latitude.

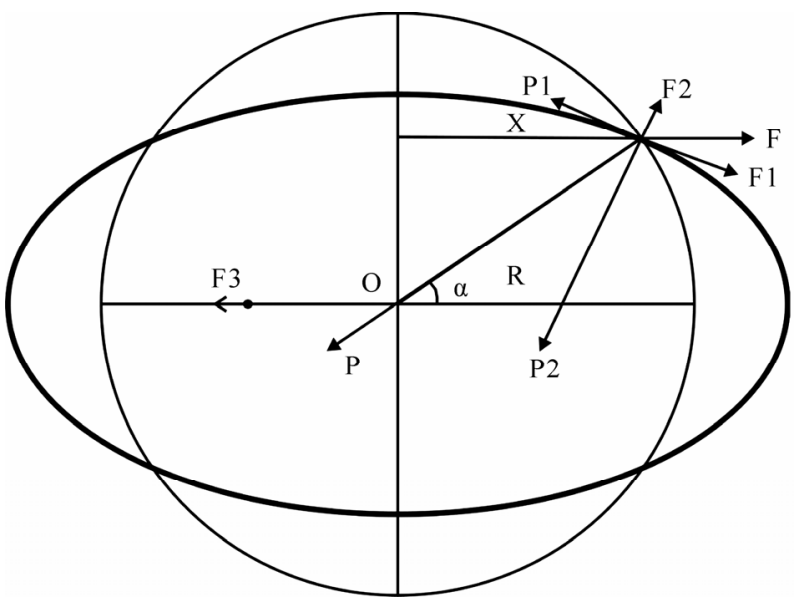

Figure 5. Forces associated with the change of the earth rotation. X-the distance between point $\mathrm{A}$ and the rotation axis of the earth, O-the core of the earth, R-radius of the earth, $\alpha$-latitude, F-extraneous centrifugal force, F1-longitudinal constituent of the extraneous centrifugal force, F2-radial constituent of the extraneous centrifugal force, F3-extraneous latitudinal force, P-gravity, P1-longitudinal constituent of the gravity, P2-radial constituent of the gravity.

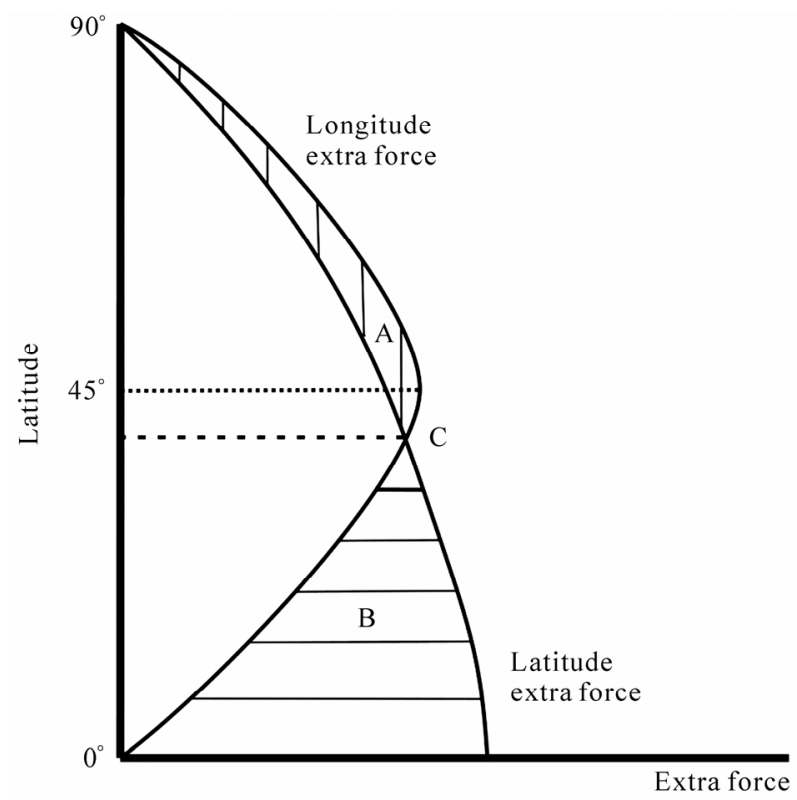

Figure 6. The distribution of extra forces in latitude and longitude.

For a terrace with a unit volume, the F3 can be donated by the following

$$
F 3=\rho(\tau-1) \frac{d w}{d t} \cdot R \cdot \cos \alpha
$$

where the $\rho$ is density. There will be action force between two terraces with different mass or different density.

The Coriolis force comes into being because rotation 
line speed differs in different latitudinal area. For example, mobile air can change heading affected by this force. In view of the deduction, when the mantle moves from the deep to the Earth's surface or from low latitude to high latitude, it is affected by the Coriolis force due to speed changing. And the mantle flow will change movement direction.

\section{RELATIONSHIP}

It is assumed that magnitude of tectonic stress caused by alteration of the earth rotation rate is $10^{-2}$ to several $\mathrm{Pa}$, which is much less than magnitude of tectonic stress actually measured from ancient to modern [5,27], hence the force relating to the earth rotation inertia force fails causing lithosphere plate to break or move.

Probability of mantle convection and gravitation relating to the mantle convection are enough to cause breaking and movement of the plate. The associated forces with the mantle rising and the plate movements include gravity and drag forces related to the mantle convection and the dynamic slab of a subducting plate. Mantle convection is relevant to the earth heat. The mantle starting to move up from the core-mantle boundary or transition zone base may arouse redistribution of the earth interior substance. Thereby they cause alteration of the earth rotation angular speed, developing increment of the earth rotation inertia force. Whereas this rotation inertia force restrains and regulates the initial mantle convection, hence decides the direction of plate movement at last and in turn decides the orientation of deformation.

If the change of angular velocity $\Delta w$ is great, and the angular acceleration $\frac{d w}{d t}$ is small, the longitudinal extraneous force will be significant. And the trend of the possible mid-oceanic ridge will be parallel to latitudinal line (Figure $\mathbf{7 ( a )}$ ). When the earth rotation becomes slow, the lithosphere plates will move to the North and South Pole, and the possible mid-oceanic ridge trend will be along the equator. When the earth rotation becomes fast, the lithosphere plates will move toward the equator, and the possible mid-oceanic ridge trend will be in the north and south half globes and parallel to the latitude lines. If the angular acceleration $\frac{d w}{d t}$ is great, and the latitude extraneous force will be dominating. The upwelling mantle will prefer to grow in the longitudinal direction, and the trend of the possible mid-oceanic ridges will be along the longitudinal lines (Figure 7(b)).

If the difference between the longitudinal extraneous force and the latitude extraneous force is small, the trend

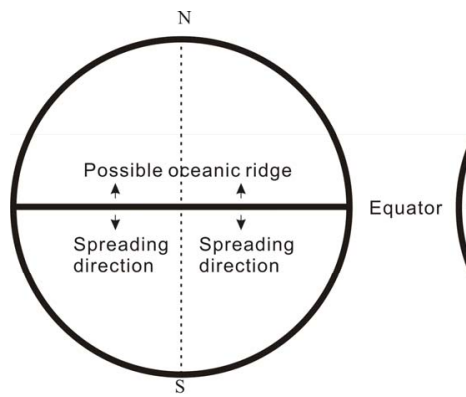

(a)

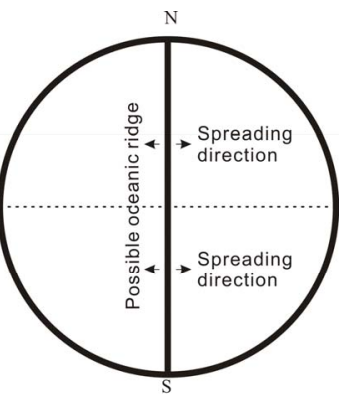

(b)
Figure 7. Possible mid-oceanic ridge trend when either longitudinal extraneous force (a) or latitudinal extraneous force (b) is dominating.

of the possible mid-oceanic ridge will controlled by both of the two forces, and it will be along the "possible mid-oceanic ridge" in Figure 8. The line of the possible mid-oceanic ridge can be divided into five segments. The segment DD' is nearly parallel to the longitudinal line, and parallel to the longitudinal line in the equator where the longitudinal extraneous force is zero. The segment DC or D'C' connects with the longitudinal lines, and it trends NE or NW in this case like Figure 8(a), while the earth rotation speed becomes slow. The segment $\mathrm{CN}$ or C'S concave to the west and its trend changes from NE to NNE northward in the north half globe, or from SW to SSW southward in the south globe. This is due to that the decrease in magnitude of the longitudinal extraneous force is faster than that of the latitude extraneous force as indicated in the Figure 6. The lengths of the five segments depend on the relative magnitude of the latitudinal extraneous force to the longitudinal extraneous force. When the latitudinal extraneous force is bigger than the longitudinal extraneous force, the segment DD' will become long and the other segments will become short. The trend of the possible mid-oceanic ridge will parallel to the longitudinal line. When the latitudinal extraneous force is less than the longitudinal extraneous force, the segment DD' will become short and the other segments will become long. The trend of the possible mid-oceanic ridge will be NE or SW.

In the case that the earth rotation becomes fast, the lithosphere plates will move toward the equator, the shape and the relationship of the trend of mid-oceanic ridge to longitudinal line do not change (Figure $8(\mathbf{b})$ ). The trend of mid-oceanic ridge line will in the east half globe and symmetrical to the trend of mid-oceanic ridge in Figure 8(a).

Although the force related to the earth rotation can not break or move a plate, it can control the growing trend of an upwelling mantle. In turn, it controls the directions of 


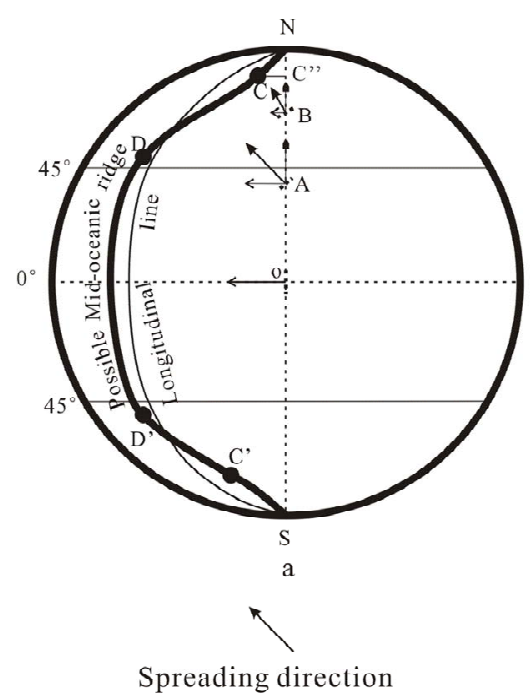

$\leftarrow$

Latitudinal extraneous force

(a)

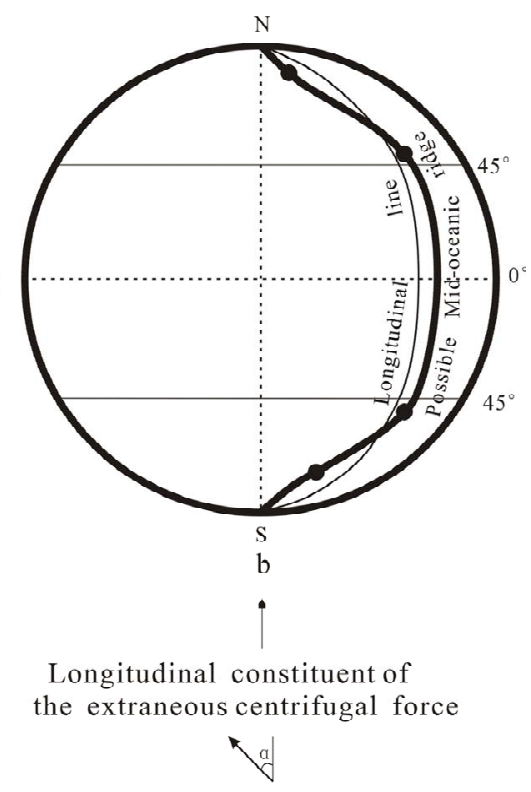

$\alpha$ : the angle between the spreading direction and the longitudinal line

(b)

Figure 8. Possible trend of the mid-oceanic ridge when the magnitude difference between the longitudinal and latitudinal extraneous forces is small. (a)the earth rotation velocity becoming slow, (b)the earth rotation velocity becoming fast. Given the sense of the latitudinal extraneous force is westward. The longitudinal line is of no longitude value, which is applied here to indicate its relationship to the trend of mid-oceanic ridge. The $\alpha$ becomes big from point $\mathrm{O}$ to certain point $\mathrm{C}$ " in the line of NS which matches the point $\mathrm{C}$ along latitudinal line. From point $\mathrm{C}$ " northward, the $\alpha$ becomes small.

plate movements for the plates move perpendicular to the mid-oceanic ridge and parallel to the transform faults. Regarding the plates in the north half globe or in the south half globe, the trends of possible mid-oceanic ridges may be parallel to the longitudinal lines, latitudinal lines or may trend northeast or northwest, depending on both the relative magnitude of longitudinal extraneous force to latitudinal extraneous force and the change of the earth rotation velocity.

\section{DISCUSSION}

The established models of mid-oceanic ridges (Figure 8) match well with the distribution and trends of present mid-oceanic ridges (Figure 1). The Atlantic mid-oceanic ridge and the east branch of the Indian mid-oceanic ridge are identical to the possible mid-oceanic ridge in Figure 8a. The changes of the trend of mid-oceanic ridges near the equator are resulted from the disharmonic deformation between the north half globe and the south half globe [7] or are related to the Coriolis force, which caused the north half globe move westward relative to the south half globe [20]. The geometric shape of the Pacific mid-oceanic ridge is similar to that of the possi- ble mid-oceanic ridge in Fig.8a, but the relationship of the Pacific mid-oceanic ridge to the longitudinal lines is different from that in the Figure 8(a). If the Pacific mid-oceanic ridge is rotated by an angle of $20^{\circ}$ clockwise, it will nearly parallel to the Atlantic mid-oceanic ridge, and will match the possible mid-oceanic ridge in Figure 8(a). This indicates another place of the earth rotation axis when the Pacific mid-oceanic ridge formed. In other word, it can be deduced that the earth rotation axis drifts from the formation of the Pacific mid-oceanic ridge. The west branch of the Indian mid-oceanic ridge trends northeastward, which may be affected either by the increase in the earth rotation velocity or by the northward movement of the all plates of the earth due to the Africa superwell [28].

Furthermore, the extensive activities of the plate tectonics are sound related to the changes of the earth rotation speed (Figure 9). The hotspots in Hawaiian Islands show that the Pacific plate moved toward north from Paleocene to Eocene, and moves toward NWW or W from Oligocene to the present [28]. The moving direction change corresponds to the change of the earth rotation speed from a slow decrease to a fast decrease (Figure 9). The longitudinal extraneous force dominated for 
the former, and the latitudinal extraneous force dominated for the latter. The two forces prefer to form east-west trend mid-oceanic ridge and north-south trend mid-oceanic ridge, respectively. The corresponding plate movements are toward the north or west, respectively. These match the viewpoints indicated in the Figure 7.

Some other problems should be noted. Where one plate collides with another plate, their movement directions will change. As a result, the deformation direction will change depending on the collision and the geometric shape of the plates. In the models like Figure 7 and Figure 8 , if the Coriolis force and some other force relating to the earth rotation are accounted, the mantle movement situation will be more complex. For example, when a mantle plume, which has considerable impact on tectonic evolution of plates [25], moves from the deep to shallow, it will deflect westward because line speed in shallow layer is greater than that in deep layer.

In fact, these forces associated with the earth rotation operate connectively at the same time. This will cause complex pictures of plate movements at various latitude and longitude. In turn, this will lead complicated crust deformation assemblages like what can be seen today. It is worth further study.

Finally, on deciding plate movement, relationship between mantle convection and the earth rotation can be represented with a carton model (Figure 10), called the

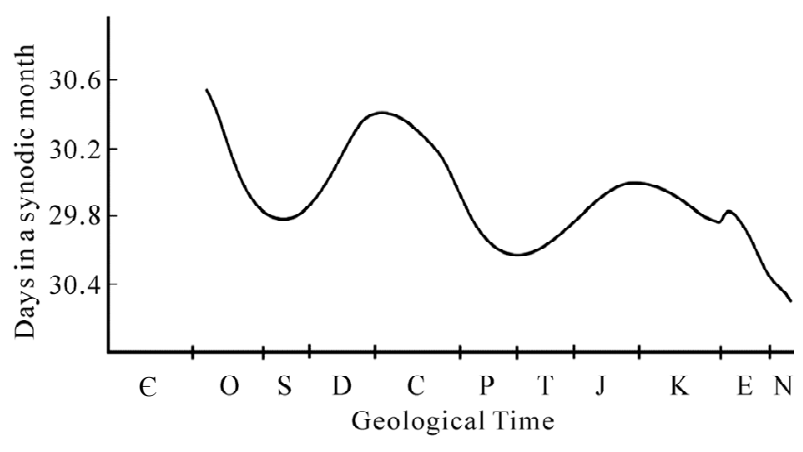

Figure 9. The change of the earth rotation speed in the Phanerozoic (Modified after reference [20]).

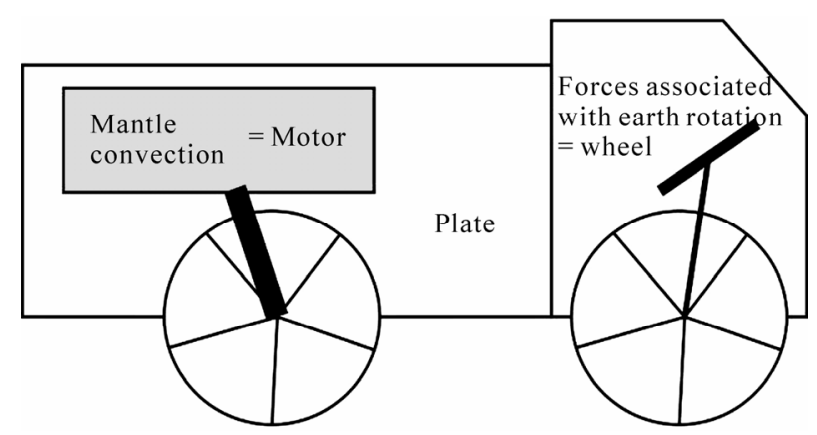

Figure 10. Carton of the Earth Dynamic Car.
"Earth Dynamic Car". In this dynamic system, the mantle convection is the power source, accounting the engine of one car and being the power source of the plate break and movement. While the force relating to the earth rotation accounts the steering wheel of the car, restraining the direction of the plate movement.

\section{CONCLUSION}

The earth basic dynamic system should include the content in two aspects, namely the mantle convection, relating to the earth heat and gravity, and the earth rotation. The mantle convection is the power source of the plate break and movement, while the earth rotation restrains the growing trends, and in turn controls the direction of the plate movement. Above both are two parts of dynamic system that can not be separated. The model connects the thermal and the rotation of the earth.

\section{ACKNOWLEDGEMENTS}

The author thanks Prof. Ma Zongjin and Prof. Jin Zhijun for their guidance on his research work and creative suggestions in the preparation of this paper.

\section{REFERENCES}

[1] Lee, J.S. (1979) The Method of Geomechanics. Scientific Press, Beijing.

[2] Lee, J.S. (1994) Introduction to Geomechanics. 2nd Edition, Geological Publishing House, Beijing.

[3] Ma, Z.J., Du, P.R., Ren, J.W. and Gao, X.L. (2006) Earthquake episodes in the low latitude global shear zone of the earth and the circle of the Pacific. Science in China (Series D: Earth Science), 36, 326-331.

[4] Wu, Z.H. and Cui, S.Q. (1995) Direction and equidistant distribution of mid-ocean ridges and the mechanism of their formation. Journal of Geomechanics, 1, 15-24.

[5] Ma, Z.J. and Du, P.R. (1995) The problems on recent crustal movement. Geological Publishing House, Beijing.

[6] Ma, Z.J., Zhang, P.Z., Ren, J.W., Feng, R. and Zhang, J. (2003) New cognitions on the global and Chinese continentental crustal vector fields. Advance in Earth Sciences, 18, 4-11.

[7] Ma, Z.J., Ren, J.W. and Zhang, J. (2003) Global tectonics and the plate motion obtained from the ITRF97 station velocity vectors. Science in China (Series D: Earth Science), 46, 193-199.

[8] Ma, Z.J., Du, P.R. and Hong, H.J. (2003) Structure and Dynamics of the earth. Guangdong Science and Technology Press, Guangzhou.

[9] Wilson, J.T. (1965) A new class of faults and their bearing on continental drift. Nature, 207, 343-347.

[10] Morgan, W.J. (1968) Rises, trenches, great faults, and crustal blocks. Journal of Geophysics Research, 73, 8275-8291.

[11] McKenzie, D. and Parker, R.L. (1967) The north pacific: an example of tectonics on a sphere. Nature, 216, 1276- 
1280.

[12] Cox, A. and Hart, R.B. (1986) Plate tectonics: How it works. Blackwell Scientific Publications, Palo Alto.

[13] Marques. P.R. and Lourenco, C.N. (2007) Physical models of rifting and transform faulting, due to ridge push in a wedge-shaped oceanic lithosphere. Tectonophysics, $\mathbf{4 4 3 ,}$ $37-52$.

[14] Wegener. (2006) The origin of sea and continent. Peking University Press, Beijing.

[15] Wells, J.W. (1963) Coral growth and geochronometry. Nature, 197, 948-950.

[16] Bo, W.J. and Wang, G.Y. (2006) Research on relations among earth rotation, fault deformation and seismicity. Journal of Geodesy and Geodynamics, 26, 43-47.

[17] Song, G.Y., Cao, Z.C., Wang, J.Y. and Yang, T.L. (2007) The annual variation of the earth's rotation speed. Progress in Geophysics, 22, 1225-1228.

[18] Chen, S.L., Zhang, H., Zhu, G.Z. and Shi, Y.L. (2008) Global displacement and stress fields induced by fast change of Earth's angular velocity. Earth Quake, 28, $1-12$.

[19] Yang, G.A. and Shi, J. (2009) Testing for the granger causality of the changes of the Earth's rotation and global earthquakes of magnitude above 5.0. Earth Science Frontiers, 16, 378-383.

[20] Gao, Q.H. (1996) The problems on crustal movement. Geological Publishing House, Beijing.
[21] Ma, Z.J., Ren, J.W. and Zhang, J. (2003) The present plate movements shown by GPS vector fields and the model of the combined latitudinal and longitudinal mantle flow. Earth Science Frontiers, 10, 5-13.

[22] Heirtzler, J.R., Dickson, G.O., Herron E.M., Pitman, W.C. and Pichon, X.L. (1968) Marine magnetic anomalies, geomagnetic field reversals and motions of ocean floor and continents. Journal of Geophysics Research, 73, 2119-2135

[23] Davis, G.F. (1999) Dynamic Earth. Cambridge University Press, Cambridge.

[24] Allen, P.A. and Allen, J.R. 1990. Basin analysis: Principles and application. Blackwell Scientific Publications, Oxford.

[25] Burov, E., Frottier, L.G, d'Acremont, E., Pourhiet, L.L. and Cloetingh, S. (2007) Plume head-lithosphere interactions near intra-continental plate boundaries. Tectonophysics, 434, 15-38.

[26] Turcotte, D.L. and Schubert, G. (2002) Geodynamics. John Wiley and Sons, New York

[27] Wu, Z.H. (1997) Geodynamics of the rotating earth. Geological Publishing House, Beijing.

[28] Pasyanos, M.E. and Nyblade, A.A. (2007) A top to bottom lithospheric study of Africa and Arabia. Tectonophysics, 444, 27-44.

[29] Wilson, J.T. (1973) Mantle plumes and plate motions. Tectonophsics, 19, 149-164. 